\title{
Brief Review of Liquid Crystals
}

\author{
V. Viswanatha ${ }^{1}$, C. Rajaramb ${ }^{2}$, S. R. Fathimac ${ }^{3}$, D. Bhanu priyad ${ }^{4}$ \\ ${ }^{1}$ Assistant Professor, ${ }^{2,3,4}$ Associate Professor \\ ${ }^{1,3,4}$ Department of Pharmaceutics, ${ }^{2}$ Department of Pharmacology \\ P. Rami Reddy Memorial Collage of Pharmacy, Kadapa, Andhra Pradesh, India
}

\begin{abstract}
Liquid crystal (LC) is the substances that flow like liquids but maintain some of the ordered structure characteristics of liquid crystals. Examined and summarized the types of liquid crystals. Analyzed the structural characteristic of smectic, nematic and cholesteric liquid crystals. It is noted that cholesteric liquid crystals are helically twisted structure and the pitch of the helix which is temperature dependent and individual chemical properties of liquid -crystalline compounds of cholesterol, Results on the influence of temperature on rheological properties of cholesteric liquid crystal are presents. This chapter describes briefly about classification of liquid crystals their structure and properties. the main aim of this review article is to scientifically highlight pharmaceutical liquid crystals, its importance in current development of targeted drug delivery system. Objects of this review is also to provide in depth information of pharmaceutical liquid crystal technology which include advanced technology development.
\end{abstract}

Keyword: Pharmaceutical liquid crystals, history, Liquid crystal displays, pharma and non-pharma applications, structures, Drug delivery utilizing LCs.

\section{INTRODUCTION}

Liquid crystals (LCs) are matter in a state which has properties between those of conventional liquids and those of solid crystals. For example, a liquid crystal may flow like a liquid, but its molecules may be oriented in a crystal-like way [1]. These are many different types of liquid phases, which can be distinguished by their different optical properties (such as texture). The contrasting areas in texture to domains where the liquid - crystals molecules are oriented in different directions. Within a space, however the particles are well ordered. LC materials may not generally be in a liquid -crystal phase (similarly as water may turn into ice or steam). Liquid crystals can be divided into thermo tropic, lyotropic and met allotropic phases. Thermo tropic and lyotropic liquid crystals consist mostly of organic molecules, although a few minerals are also known. Thermo tropic LCs exhibit a phase transition into the liquid crystal phase as temperature is change. Lyotropic LCs exhibit phase transition as a function of both temperature and concentration of the liquid crystal molecule in solvent (typically water). Met allotropic LCs are compounds of both organic and inorganic; their liquid crystal transition depends not only on the inorganic and organic ratio [2]. Example of liquid crystals can be found both in the natural world and in technological applications. Most contemporary electronic displays use liquid crystals. Lyotropic liquid crystalline phases are abundant in living systems but can also be found in the mineral world. For example, many proteins and cell membrane are liquid crystals. Other well known examples of LCs are solutions of soap and various related detergents, as well as the tobacco mosaic virus, and some clays [3]. 


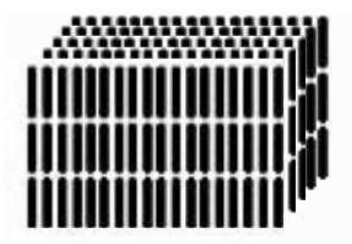

Crystalline, solid
phase

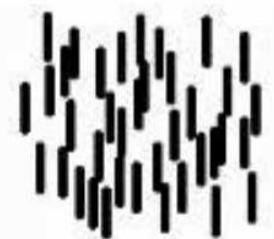

Liquid crystal phase

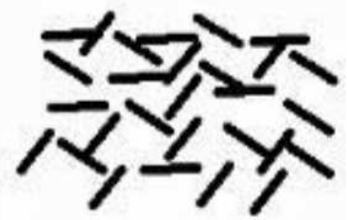

Liquid amorphous phase

Cooling Heating

Crystals:

3D long range order $(\infty$ position- and orientational order)

\author{
Mesophases / liquid crystalline phases: \\ orientational order, \\ translational long range order \\ in 1 or 2 dimensions
}

Liquids:

No long range order,

Statistical arrangements of molecules

\section{Historical background:}

$>$ 1849: W. Heintz found that upon heating ( $\mathrm{t}$ (at 52c), stearin, softened and become cloudy, and at 62.5 it becomes clear. Duffy partrayed this phenomenon as the presents of two melting points

$>$ 1950: Mettenheimier found myelin to be birefringent, a property previously known to be possessed by crystals only

$>$ 1888: Reinitzer observed the two -melting-points phenomenon, birefringence and the occurrence of iridescent colors between the two melting points in a material that we now was cholesteryl benzole

> 1889: Lehmann carried out detail tail investigation and reasoned that the birefringent portions of the liquid must be crystals. He previously referred to this material as liquid crystals
1890: Gattermann blended the principalfluid precious crystals of azoxybenzene, with fully known structures

1908: D. Vorlaender, the first to integrate a thermotropic smectic compound, related liquid crystallinity to chemical structures, detected polymorphism of liquid crystal state

1922: Freidel identified and named thedifferent microscopic textures (nematic, smectic, and cholesteric), observed the impacts of electric and magnetic fields

1930: Freedericksz studied the transition from a homogeneoustructure at some critical valuer of applied field strength

1965: the global liquid crystal society by late Glenn Brown at kent state university. Lehmann and Reinitzer are known as the grandfathers of LCs science [4-5]

\section{Classification:}
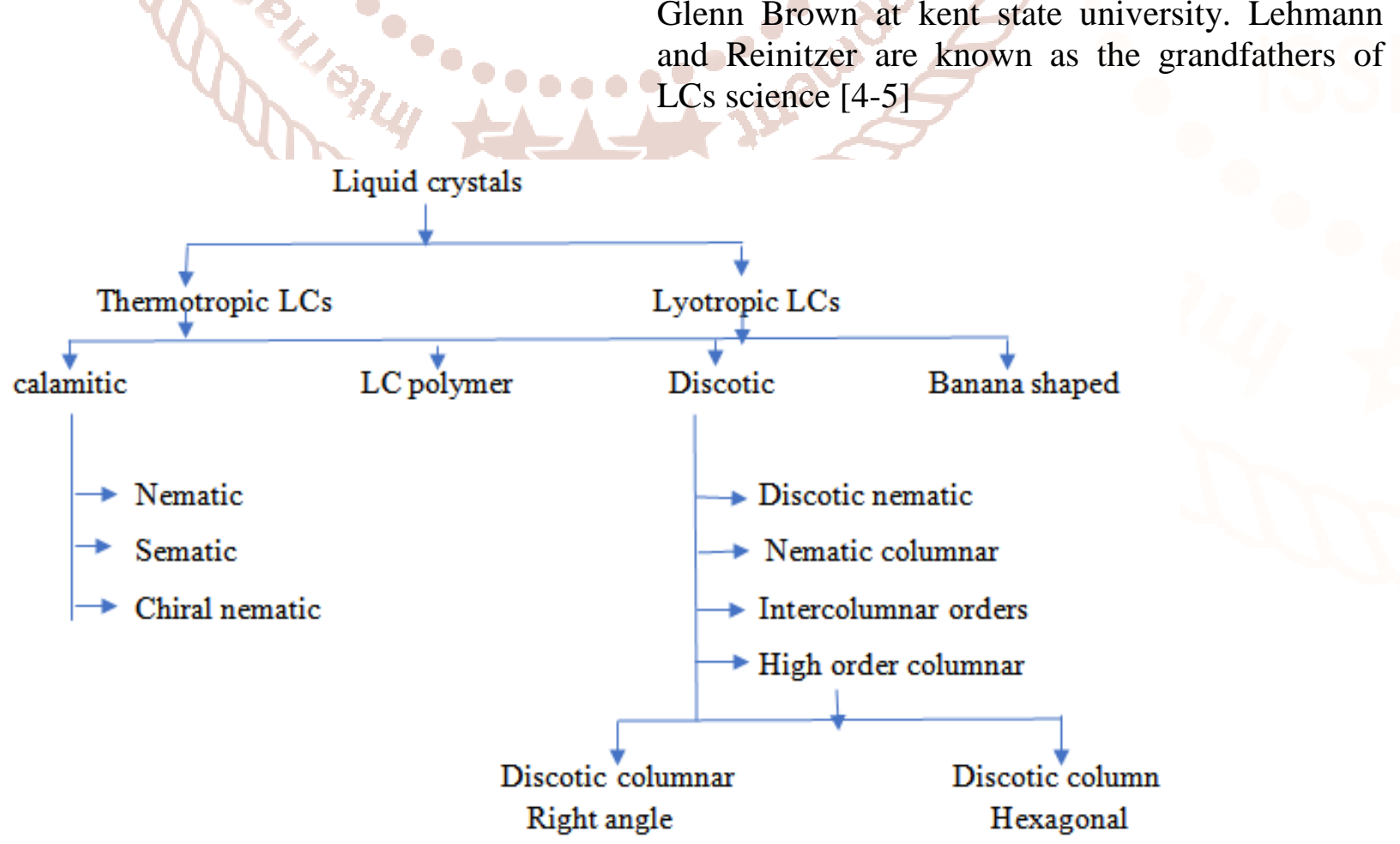
Chemical structure: All the known molecules that Monomers commonly used: form liquid crystalline phases are asymmetric in their shape.

Monomers can be attached: to in polymer back bone forming side chain polymer
$>$ Cholesterol ester

$>$ Phenyl benzene

$>$ Surfactant

$>$ a long single chain forming main chain polymer

$>$ Paraffines

$>$ Glycolipids

$>$ Cellulose derivatives[6]

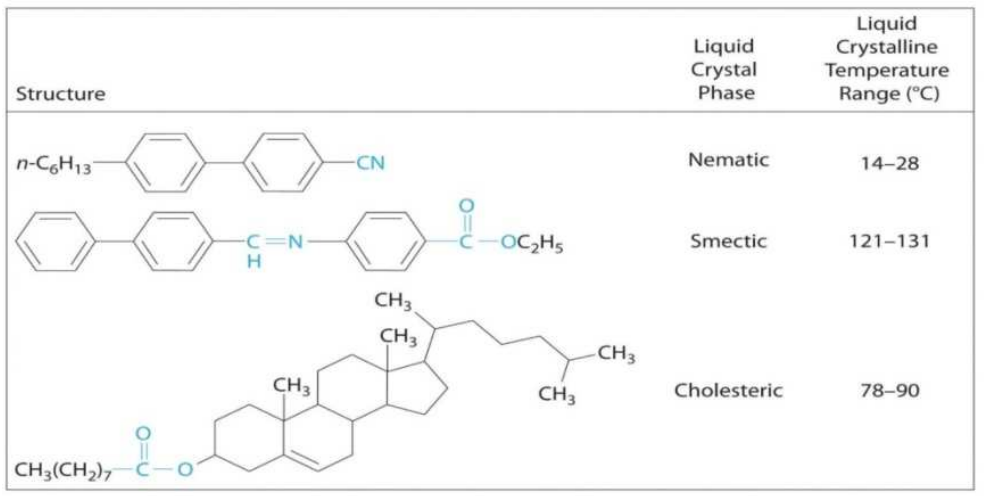

Characteristic features and properties:

$>$ point along a common axis, the director

$>$ Orientational arrange is between the customary strong and fluid stages

$>$ Mesogens Anisotropic i.e. the properties very contingent upon what course they are estimated with respect to the chief

$>$ Birefriengent, resulting in the two components of electric or magnetic field travelling through it at different speed hence out of phase when they exit

> Stiffness necessary for liquid crystallinity results from restrictions on rotation caused by steric hindrance and

> Axial proportion $(\mathrm{x}=\mathrm{l} / \mathrm{d})$ must be something like three to display the attributes of liquid crystals

\section{Properties:}

$>$ Liquid crystal can flow like a liquid, due to loss of positional order

$>$ Liquid crystal is optically birefringent, due to its orientational order

Transition crystalline solid to liquid crystals caused by a change of temperature - given rice to THRMOTROPIC liquid crystals

$>$ Substances that are most likely to form a liquid crystals phase at a certain temperature are molecules that are ELONGATED \& have some degree of rigidity [7].

\section{Temperature range problems:}

Methods of lowering the polymer melting temperature:

Minimized interactions with reduced symmetry

$>$ Introduction of defects to disrupt the ability for neigh boring polymers to line up

Molecules are assembled in arbitrary orientation (head to tail, head to head)

Random copolymerization result in irregularity of polymer substituents

\section{LCs Display:}

A liquid crystal display (LCD) is a flat -panel display or other electronically regulated optical device that uses the light -tweaking properties of liquid crystals. Liquid crystals do not emit light display, instead using a backlight or reflector to produce image in color or monochrome [8-9].

$>$ LCs consists of an array of tiny segments (called pixels) that can be manipulated to present information

LCD consist primary of two glass plates with some liquid crystals material between them[10] LIQUID CRYSTAL DISPLAY

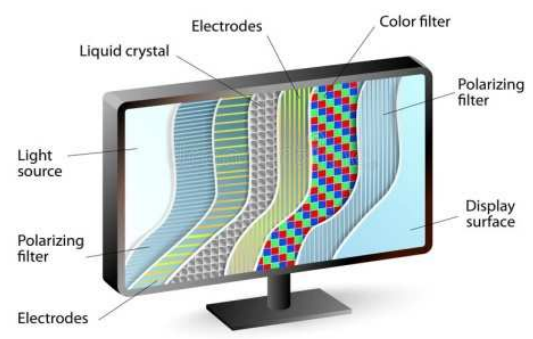


$>$ Plates are usually manufactured with transparent electrodes (ITO), that make it possible to apply an electric field across small areas of the film of liquid crystal

$>$ LCDs consume much less power than their cathodes -ray tube (CRT) counterparts

$>$ Most usually utilised are the twisted nematic (TN) Displays[11]

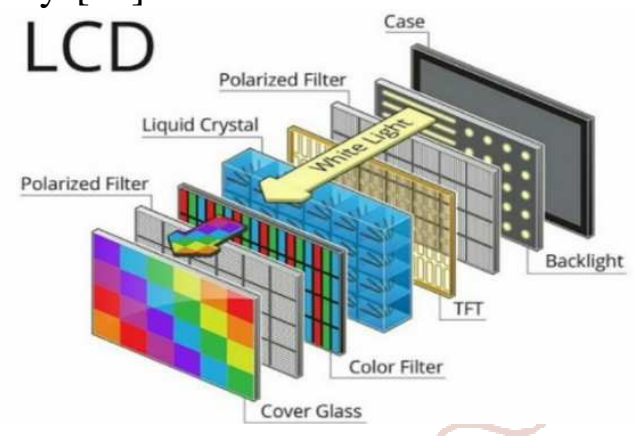

Advantages:

$>$ The liquid crystal display (LCDs)

$>$ Liquid crystals thermometers. Cholesteric liquid crystals reflect light with a wavelength equal to the pitch which is dependent upon temperature

$>$ High strength fibers. Polymer liquid crystals are used in applications calling for strong, lightweight materials e.g. Kevlar

$>$ Optical imaging. Generation of electric field as light falls on photoconductor plates

$>$ Drug delivery. Lyotropic liquid crystals can coat a drug to keep it from being destroyed in the digestive tract

$>$ Biological membrane. Lyotropic liquid crystals act as biological membranes due to their amphiphilic nature [12].

\section{Latest Development in Pharmaceutical Liquid Crystal Technology:}

Kent state university, Summa health system and IC MedTech have developed a new paradigm in drug discovery based on the pharmacologic properties of liquid crystal pharmaceuticals (LCs). LCPS are a unique class of lyotropic liquid crystals that represent novel drug candidates for a treatment of a wide range of diseases. Representatives of this research project recently filed applications for two new patients: one for a new LCP-based anti -tumor drug called TOLECINE and another for a formulation that combines TOLECINE and another LCP, APATONE.

Olecine is a new pre-clinical anti-tumor LCP that also has antiviral and antibacterial applications. It is tumor- cell selective and exhibits a strong antineoplastic activity (it counteracts abnormal proliferation of cells in a tissue or organ). Likewise, it has been appeared to be more visable than the present standard of condition for herpes.

Apatone is a clinical phase investigational new drug for late- stage prostate cancer. It is currently under consideration for clinical study for potential applications such as augmentation of chemotherapy to allow lower, less toxic doses of common chemotherapy agents. Apatone is made from two non -toxic compounds, a liquid crystal compound and a sugar, that selectively bio-concentrate with in a cancer cell and produce a free radical. Formation of strong, short lived free radical is a concentration driven intracellular reaction and therefore only takes place in cells with sufficient sugar concentrations (such as cancer cells). The reaction results in oxidative stress that weakens the targeted cells from within. It is carried out quickly only within the cancer cells, with no toxic reaction by products that might harm adjacent healthy cells [13].

\section{Pharma drugs as LCS:}

Many little atomic pharmaceutical dynamic compounds have been shown to frame LC meso phases. Nafoxidine hydrochloride is one such example; this cationic drug has amphiphilic properties and gives rice thermo tropic (smectic type) and lyotropic liquid crystalline structures. Palmitolyl propranolol hydrochloride is an amphiphilic derivative of beta-blocker propranolol hydrochloride which forms smectic type liquid crystalline phase. It has been administered as liquid crystalline dispersion for cardiac problems. Itraconazole hydrochloride is an antifungal medication which frames chiral nematic stages. Some of the other examples of small molecular pharmaceuticals which can from LCs are arsphenamine, fenoprofen sodium, fenoprofen calcium, penbutolol sulphate, nafcillin, methotrexate, folic acid and to bramycin. Large molecular pharmaceutical active compounds are also known to form LCs; some common examples of them are cyclosporin, calcitonin, amylin, nafarelin, detirelix, and leuprolide. The recent research has yielded a new investigational LC based anti-tumor drug called tolecine, a compound which also has antiviral and antibacterial applications. Some of the pharmaceutical excipients such as hydroxy propyl cellulose, ethyl cellulose and cellulose acetate have also displayed LC phases. Besides this, examples of naturally occurring LCs are DNA, cholesterol and the biological membranes [14]. 
Drug delivery utilizing liquid crystal structure:

Vitamin E TPGS/drug compositions and methods are provided which abviate the need for surfactants or non-evaporated co solvents because the active drug component is dissolved directly onto vitamin E TPGs to form a true molecular solution -not an emulsion or a micro emulsion. The innovation gives a gradually dissolving TPGS/sedate framework that ingests gastrointestinal liquid into the grid at the measurement shape/liquid interface, where a gel like liquid crystal is formed [15]. This gel front structures a liquid crystal limit where tranquilize disintegration is most. At this liquid crystal/GI fluid boundary, synchronization takes place in which the rare of formation of liquid crystal equal the dissolution rate of liquid crystals at the water interface, thereby giving controlled order release of the drug into the GI tract[16]. The rate of disintegration is additionally controlled by the geometry of the measurement shape. The solid vitamin E TPGS/drug matrices of the invention can be solidified and compressed into tablet or filled into capsules, with other excipients, binders and / or fillers [17]. The solid TPGS/drug solution of the invention also can be made into an immediate release liquid formation upon addition of water, or into a controlled release system liquid formation up on addition of water, or into a controlled release system solid tablet by the use of impermeable or semi permeable barrier or coatings surrounding portions of the tablet [18].

\section{Applications in pharma:}

It has been estimated that approximately 5 percent of all organic molecules are able to exist as temperature LCs. Pharmaceutical compounds have been increasingly characterized by their lyotropic liquid crystalline states with relatively fewer examples of thermo tropic LC states. LLCs based delivery system such as creams, ointments, gels, liposomes, colloidal dispersions and transdermal patches have been used in pharmaceuticals and cosmetics.

\section{Non-Pharma applications of liquid crystals:}

$>$ Liquid crystal displays

$>$ Liquid crystal thermometers

$>$ Optical imaging

Liquid crystal technology has had a major effect many areas of science and engineering, as well as device technology. Applications for this special kind of material are still being discovered and continue to provide effective solution to different problems.
Liquid crystals have a multitude of other uses. They are used for non-destructive mechanical testing of materials under stress. This strategy is likewise for the perception of RF (radio recurrence) waves in waveguides. They are used in medical applications where, for example, transient pressure transmitted by a walking foot on the ground is measured. Low molar mass (LMM) liquid crystal have applications including erasable optical disks, full color "electronic slides" for computer- aided drawing (CAD), and light modulators for color electronic imaging.

As a new properties and types of liquid crystals are investigated and researched, these materials are sure to gain increasing importance in industrial and scientific application [19].

\section{Conclusion:}

There are three states of matter -gas liquid and solid. A fourth condition of issue is the fluidcrystalline state. The term liquid crystal is an apparent contradiction, but it is useful in a descriptive sense because material in this state are in many ways intermediate between the liquid and solid states. The liquid crystalline state may result either from the heating of solids or from the action of certain solvents or solids (lyotropic liquid crystals). LLCs based delivery systems such as creams, ointments, gels, liposomes, colloidal dispersions and transdermal patches have been used in pharmaceuticals and cosmetics. Liquid crystals method for sedate conveyance can be a viable and helpful for conveying the medication with wanted target. This technique can be utilized largely in topical delivery of the drug as it passes the advantages of smooth feel and drug loading of incompatible molecule. Day by day various patients are coming in this liquid-crystals technique. This technique of drug loading is a new and immerging techniques requires attention further for its practice in real scientific industries to deliver a quality outcome for a society. Non -pharma applications LCs are LCS displays, LCS thermometers and optical imaging. As research into this field continues and as new applications are developed, liquid crystals will play a vital role in modern technology.

\section{References:}

1. Collings, P. J. \& Hird, M. (1997). Introduction to Liquid Crystals. Bristol, PA: Taylor \& Francis. ISBN 0-7484-0643-3. 
2. Priestley, E. B.; Wojtowicz, P. J. \& Sheng, P. (1974). Introduction to Liquid Crystals. Plenum Press. ISBN 0-306-30858-4.

3. Denis Andrienko, "Introduction to liquid crystals "International Max Planck Research School Modelling of soft matter 11-15 September 2006, Bad Marienberg, September 14, 2006:27-29.

4. Sluckin, T. J.; Dunmur, D. A. \& Stegemeyer, H. (2004). Crystals That Flow - classic papers from the history of liquid crystals. London: Taylor \& Francis. ISBN 0-415-25789-1.

5. Hirohisa Kawamoto (2013), The history of liquidcrystal display and its industry, HISTory of ELectro-technology CONference (HISTELCON), 2012 Third IEEE, Institute of Electrical and Electronics-

Engineers,DOI10.1109/HISTELCON.2012.64875 87.

6. Reinitzer, Friedrich (1888). "Beiträge zur Kenntniss des Cholesterins". Monatshefte für Chemie (Wien). 9 (1): 421-441. Gray, G. $W$. (1962) Molecular Structure and the Properties of Liquid Crystals, Academic Press

7. Tomczyk, W.; Marzec, M.; Juszyńska-Gałazka, E.; Wegtowska, D. (2017). "Mesomorphic and physicochemical properties of liquid crystal mixture composed of chiral molecules with per fluorinated terminal chains". Journal of Molecular Structure. 1130: 503-510. Bibcode:2017JMoSt1130.503T. Doi:10.1016/j.molstruc.2016.10.039.

8. U. S. Patent 3,834,794: R. Soref, Liquid crystal electric field sensing measurement and displaydevice, filed-June28, 1973.

9. U. S. Patent 5,598,285: K. Kondo, H. Terao, H. Abe, M. Ohta, K. Suzuki, T. Sasaki, G. Kawachi, J. Ohwada, Liquid crystal display device, filed Sep 18, 1992 and Jan 20, 1993.

10. Competing display technologies for the best image performance; A. J. S. M. de Vaan; Journal of the society of information displays, Volume 15, Issue 9 September 2007 Pages 657-666; http://onlinelibrary.wiley.com/doi/10.1889/1.2785 199.

11. Castellano, Joseph A. (2005). Liquid Gold: The Story of Liquid Crystal Displays and the Creation of an Industry. World Scientific Publishing. ISBN 978-981-238-956-5.

12. Wang L, Huang D, Lam L, Cheng Z (2017). "Bowlics: history, advances and applications". Liquid Crystals Today. 26 (4): 85-111. doi:10.1080/1358314X.2017.1398307.

13. Ganesh shete, Vibha puri, Arvind K Bansal, "Liquid crystals in pharma", India's most comprehensive portal-Pharmabiz, March.25, 2009.

14. Chandrasekhar, S. (1992). Liquid Crystals (2nd Ed.). Cambridge: Cambridge Press. ISBN 0-521-41747-3.

15. P. V. Patel, J. B. Patel, R. D. Dangar, K. S. Patel, K. N. Chauhan, "Liquid Crystal Drug Delivery System”,Int.J.ofPharma.\&App.sci./1(1)/2010:118123

16. Garry Myers, Kingsport, Tenn. "Drug delivery system utilizing liquid crystal structure" US 5,891,845/ April 6,1999

17. Christel C. Mueller-Goymann, "Drug Delivery: Liquid Crystals in" Encyclopedia of Pharmaceutical Technology, Chapter 75, Third Edition Informa Healthcare

18. Imran K. Tadwee, Sourabh Gore, Prashant Giradkar "Advances in Topical Drug Delivery System: A Review” Int. J. of Pharm. Res. \& All. Sci.2012; Volume 1, Issue 1, 14-23

19. C. L. Khetrapal; R. G. Weiss; A. C. Kumar, in Liquid Crystals- Applications and uses. Vol.2, World Scientific, Singapore, 1991, p.225. 